\title{
On a Mixed Problem for Navier-Stokes System in the Unit Cube
}

\author{
N. Aliev, Sh. Rezapour, And M. Jahanshahi
}

Abstract. Mixed problems on the unit balls have special complexity. By using a new method we shall give some sufficient conditions for existence of solutions of the Fefferman's problem B ([4]).

\section{INTRODUCTION}

There are usually mathematical models based on differential equations, integral equations and integro-differential equations for physical and natural events. These models are frequently based on Cauchy problem, boundary value problem or mixed problem $([5-9,18-20])$. If there is the time variable in these equations, for verification of their solutions we obtain a boundary value problem which is depend on complex parameter by using the method in [15] or Laplace transform (see [7, 18]).

The boundary value problem may be in a bounded or unbounded region. We must provide boundary conditions in bounded regions, but solutions of the problems and their derivatives are periodic or tend to zero at infinity in unbounded regions.

The potential theory is useful in many boundary value problems, for example Dirichlet and Neumann problems. But, potential theory is not efficient in solving of many another problems (see [1-3, 10, 12-14] and [17]). We shall give a method that it will be efficient than the potential theory.

The Euler and Navier-Stokes equations describe the motion of a fluid in $\mathbb{R}^{n}(n=2$ or $n=3)$. These equations are to be solved for an unknown velocity vector $u(x, t)=\left(u_{i}(x, t)\right)_{1 \leq i \leq n} \in \mathbb{R}^{n}$ and pressure $p(x, t) \in \mathbb{R}$, defined for position $x \in \mathbb{R}^{n}$ and time $t \geq 0$. We restrict attention here to incompressible fluids filling all of $\mathbb{R}^{n}$. The Navier-Stokes equations are then given by:

2000 Mathematics Subject Classification. Primary: 35A99.

Key words and phrases. Navier-Stokes equations, Potential Theory, Boundary Conditions, Unit Ball. 


$$
\frac{\partial u_{i}}{\partial t}+\sum_{j=1}^{n} u_{j} \frac{\partial u_{i}}{\partial x_{j}}=\nu \Delta u_{i}-\frac{\partial p}{\partial x_{i}}+f_{i}(x, t), \quad\left(x \in \mathbb{R}^{n}, t \geq 0\right)
$$

$$
\operatorname{div} u=\sum_{i=1}^{n} \frac{\partial u_{i}}{\partial x_{i}}=0, \quad\left(x \in \mathbb{R}^{n}, t \geq 0\right)
$$

with initial conditions:

$$
u(x, 0)=u^{0}(x), \quad\left(x \in \mathbb{R}^{n}\right) .
$$

Here, $u^{0}(x)$ is a given $C^{\infty}$ divergence-free vector field on $\mathbb{R}^{n}, f_{i}(x, t)$ are the components of a given externally applied force (e.g. gravity), $\nu$ is a positive coefficient (the viscosity) and $\Delta=\sum_{i=1}^{n} \frac{\partial^{2}}{\partial x_{i}^{2}}$ is the Laplacian in the space variables. The Euler equations are equations (1), (2), (3) with $\nu$ is equal to zero.

Equation (1) is just Newton's low $f=m a$ for a fluid element subject to the external force $f=\left(f_{i}(x, t)\right)_{1 \leq i \leq n}$ and to the forces arising from pressure and friction. Equation (2) just says that fluid is incompressible. For physically reasonable solutions, we want to make sure $u(x, t)$ does now grow large as $|x| \longrightarrow \infty$. Hence, we will restrict to forces $f$ and initial conditions $u^{0}$ that satisfy:

$$
\left|\partial_{x}^{\alpha} u^{0}(x)\right| \leq C_{\alpha K}(1+|x|)^{-K} \quad \text { on } \quad \mathbb{R}^{n}, \quad \text { for any } \alpha \text { and } K
$$

and

(5) $\left|\partial_{x}^{\alpha} \partial_{t}^{m} f(x, t)\right| \leq C_{\alpha m K}(1+|x|+t)^{-K}$ on $\mathbb{R}^{n} \times[0, \infty)$, for any $\alpha, m, K$.

We accept a solution of (1), (2) and (3) as physically reasonable only if it satisfies:

$$
p, u \in C^{\infty}\left(\mathbb{R}^{n} \times[0, \infty)\right)
$$

and

$$
\int_{\mathbb{R}^{n}}|u(x, t)|^{2} \mathrm{~d} x<C, \quad \text { for all } t \geq 0 \quad \text { (bounded energy). }
$$

A fundamental problem in analysis is to decide whether such smooth, physically reasonable solutions exist for the Navier-Stokes equations. To give reasonable leeway to solvers while retaining the heart of the problem, Fefferman has provided four problems A, B, C and D. The authors have verified the problems $\mathrm{C}$ and $\mathrm{D}$ in [4]. Here, we restate the problem B.

(B) Existence and Smoothness of Navier-Stokes Solutions in $\mathbb{R}^{3} / \mathbb{Z}^{3}$. Take $\nu>0$ and $n=3$. Let $u^{0}(x)$ be any smooth, divergencefree vector field satisfying (8). Take $f(x, t)$ to be identically zero. Then there exist smooth functions $p(x, t), u_{i}(x, t)$ on $\mathbb{R}^{3} \times[0, \infty)$ that satisfy (1), (2), (3), (10) and (11). 


\section{On the Problem B}

Note that we could solve following problem instead the problem B:

$$
\frac{\partial u_{i}}{\partial t}+\sum_{j=1}^{3} u_{j} \frac{\partial u_{i}}{\partial x_{j}}=\nu \Delta u_{i}-\frac{\partial p}{\partial x_{i}}\left(0<x_{i}<1, i=1,2,3, t \geq 0\right)
$$

$$
\operatorname{div} u=\sum_{i=1}^{3} \frac{\partial u_{i}}{\partial x_{i}}=0, \quad\left(0<x_{i}<1, i=1,2,3, t \geq 0\right),
$$

$$
u(x, 0)=u^{0}(x), \quad\left(0<x_{i}<1, i=1,2,3\right),
$$

where $\nu>0$ and $p, u \in C^{\infty}\left(\mathbb{R}^{3} \times[0, \infty)\right)$. Also, $u^{0}$ satisfies in following condition:

$$
u^{0}\left(x+e_{j}\right)=u^{0}(x), \quad(j=1,2,3),
$$

where $\left\{e_{1}, e_{2}, e_{3}\right\}$ is the standard basis of $\mathbb{R}^{3}$. Furthermore,

$$
u\left(x+e_{j}\right)=u(x), \quad(j=1,2,3) .
$$

By using Laplace transformation on (8), (9) and (10), we have:

$$
\begin{gathered}
\Delta \tilde{u}_{i}(x, \lambda)=-\frac{\lambda}{\nu} \tilde{u}_{i}(x, \lambda)=F_{i}(x, \lambda), \quad\left(0<x_{i}<1, i=1,2,3\right), \\
\sum_{i=1}^{3} \frac{\partial \tilde{u}_{i}(x, \lambda)}{\partial x_{i}}=0, \quad\left(0<x_{i}<1, i=1,2,3\right),
\end{gathered}
$$

where

$$
\begin{aligned}
F_{i}(x, \lambda)=- & \frac{1}{\nu} u_{i}^{0}(x)+\frac{1}{\nu} \sum_{j=1}^{3} \int_{0}^{\infty} e^{-\lambda t} u_{j}(x, t) \frac{\partial u_{i}(x, t)}{\partial x_{j}} \mathrm{~d} t+ \\
& +\frac{1}{\nu} \frac{\partial}{\partial x_{i}} \tilde{p}(x, t), \quad\left(0<x_{i}<1, i=1,2,3\right) .
\end{aligned}
$$

For simplicity in notation, put $D=\left\{x \in \mathbb{R}^{3}: 0<x_{i}<1, i=1,2,3\right\}$ and $\gamma=\partial D$. It is know that fundamental solution of the Helmholtz equation $\Delta \tilde{u}_{i}(x, \lambda)-\frac{\lambda}{\nu} \tilde{u}_{i}(x, \lambda)=0$, is:

$$
U(x-\xi, \lambda)=-\frac{e^{-\sqrt{\frac{\lambda}{\nu}}|x-\xi|}}{4 \pi|x-\xi|},
$$

in the sense that:

$$
\Delta_{x} U(x-\xi, \lambda)-\frac{\lambda}{\nu} U(x-\xi, \lambda)=\delta(x-\xi) .
$$


Now, we multiple both sides of $(13)$ in $(*)$, and then we integrate the result on the region $D$. Hence, by using Gauss-Ostrogradskii formula in the first part of left hand side we have:

$$
\begin{aligned}
& \int_{0}^{1} \mathrm{~d} x_{2} \int_{0}^{1} \mathrm{~d} x_{3}\left[\left.\tilde{u}_{i}\left(1, x_{2}, x_{3}, \lambda\right) \frac{\partial U(x-\xi, \lambda)}{\partial x_{1}}\right|_{x_{1}=1}-\right. \\
& \left.-\left.\frac{\partial \tilde{u}_{i}(x, \lambda)}{\partial x_{1}}\right|_{x_{1}=1} U\left(1-\xi_{1}, x_{2}-\xi_{2}, x_{3}-\xi_{3}, \lambda\right)\right]- \\
& -\int_{0}^{1} \mathrm{~d} x_{2} \int_{0}^{1} \mathrm{~d} x_{3}\left[\left.\tilde{u}_{i}\left(0, x_{2}, x_{3}, \lambda\right) \frac{\partial U(x-\xi, \lambda)}{\partial x_{1}}\right|_{x_{1}=0^{-}}\right. \\
& \left.-\left.\frac{\partial \tilde{u}_{i}(x, \lambda)}{\partial x_{1}}\right|_{x_{1}=0} U\left(-\xi_{1}, x_{2}-\xi_{2}, x_{3}-\xi_{3}, \lambda\right)\right]+ \\
& +\int_{0}^{1} \mathrm{~d} x_{1} \int_{0}^{1} \mathrm{~d} x_{3}\left[\left.\tilde{u}_{i}\left(x_{1}, 1, x_{3}, \lambda\right) \frac{\partial U(x-\xi, \lambda)}{\partial x_{2}}\right|_{x_{2}=1-}\right. \\
& \left.-\left.\frac{\partial \tilde{u}_{i}(x, \lambda)}{\partial x_{2}}\right|_{x_{2}=1} U\left(x_{1}-\xi_{1}, 1-\xi_{2}, x_{3}-\xi_{3}, \lambda\right)\right]- \\
& -\int_{0}^{1} \mathrm{~d} x_{1} \int_{0}^{1} \mathrm{~d} x_{3}\left[\left.\tilde{u}_{i}\left(x_{1}, 0, x_{3}, \lambda\right) \frac{\partial U(x-\xi, \lambda)}{\partial x_{2}}\right|_{x_{2}=0-}\right. \\
& \left.-\left.\frac{\partial \tilde{u}_{i}(x, \lambda)}{\partial x_{2}}\right|_{x_{2}=0} U\left(x_{1}-\xi_{1},-\xi_{2}, x_{3}-\xi_{3}, \lambda\right)\right]+ \\
& +\int_{0}^{1} \mathrm{~d} x_{1} \int_{0}^{1} \mathrm{~d} x_{2}\left[\left.\tilde{u}_{i}\left(x_{1}, x_{2}, 1, \lambda\right) \frac{\partial U(x-\xi, \lambda)}{\partial x_{3}}\right|_{x_{3}=1}-\right. \\
& \left.-\left.\frac{\partial \tilde{u}_{i}(x, \lambda)}{\partial x_{3}}\right|_{x_{3}=1} U\left(x_{1}-\xi_{1}, x_{2}-\xi_{2}, 1-\xi_{3}, \lambda\right)\right]- \\
& -\int_{0}^{1} \mathrm{~d} x_{1} \int_{0}^{1} \mathrm{~d} x_{2}\left[\left.\tilde{u}_{i}\left(x_{1}, x_{2}, 0, \lambda\right) \frac{\partial U(x-\xi, \lambda)}{\partial x_{3}}\right|_{x_{3}=0-}\right. \\
& \left.-\left.\frac{\partial \tilde{u}_{i}(x, \lambda)}{\partial x_{3}}\right|_{x_{3}=0} U\left(x_{1}-\xi_{1}, x_{2}-\xi_{2},-\xi_{3}, \lambda\right)\right]+ \\
& +\int_{D} F_{i}(x, \lambda) U(x-\xi, \lambda) \mathrm{d} x= \\
& =\left\{\begin{array}{ll}
\tilde{u}_{i}(\xi, \lambda), & \xi \in D \\
\frac{1}{2} \tilde{u}_{i}(\xi, \lambda), & \xi \in \gamma,
\end{array} \quad(i=1,2,3) .\right.
\end{aligned}
$$

By above relation and lateral faces of $\gamma$, note that we could obtain six expressions which these will be our necessary conditions. By $(*)$, we have:

$$
\begin{array}{r}
\frac{\partial U(x-\xi, \lambda)}{\partial x_{m}}=\frac{e^{-\sqrt{\frac{\lambda}{\nu}}|x-\xi|}}{4 \pi|x-\xi|^{2}}\left[1+\sqrt{\frac{\lambda}{\nu}}\left(x_{m}-\xi_{m}\right)\right] \frac{x_{m}-\xi_{m}}{|x-\xi|}, \\
(m=1,2,3) .
\end{array}
$$


Remark 2.1. Note that all partial differentials of the fundamental solution, respect to $x_{m}$, calculate at $x_{m}=0$ or $x_{m}=1$ in (16). So by (17), all integrals in (16) are exist.

Now, similar to the process in [10-14], by using values of partial differentials of $\tilde{u}_{i}(x, \lambda)$ on boundary of $\gamma$, we want to obtain another necessary conditions.

Now, by multiple the equation (13) by (17) and then by integrate of both hand sides of the result on the region $D$, we obtain:

$$
\begin{aligned}
& \int_{D} \Delta \tilde{u}_{i}(x, \lambda) \frac{\partial U(x-\xi, \lambda)}{\partial x_{m}} \mathrm{~d} x- \\
& \frac{\lambda}{\nu} \int_{D} \tilde{u}_{i}(x, \lambda) \frac{\partial U(x-\xi, \lambda)}{\partial x_{m}} \mathrm{~d} x= \\
= & \int_{D} F_{i}(x, \lambda) \frac{\partial U(x-\xi, \lambda)}{\partial x_{m}} \mathrm{~d} x, \quad(i, m=1,2,3) .
\end{aligned}
$$

Note that the Gauss-Ostrogradskii formula should be use carefully. In fact, in calculates of these integrals must not appear any partial differentials of order more than two of the functions $\tilde{u}_{i}(x, \lambda)$ and $U(x-\xi, \lambda)$ in the region $D$, and any partial differentials of order more than one of these functions on the boundary of $\gamma$. Then:

$$
\begin{aligned}
& \sum_{k=1}^{3} \int_{\gamma} \frac{\partial \tilde{u}_{i}(x, \lambda)}{\partial x_{k}} \frac{\partial U(x-\xi, \lambda)}{\partial x_{m}} \cos \left(s, x_{k}\right) \mathrm{d} x- \\
- & \sum_{k=1}^{3} \int_{D} \frac{\partial \tilde{u}_{i}(x, \lambda)}{\partial x_{k}} \frac{\partial^{2} U(x-\xi, \lambda)}{\partial x_{m} \partial x_{k}} \mathrm{~d} x- \\
- & \frac{\lambda}{\nu} \int_{\gamma} \tilde{u}_{i}(x, \lambda) U(x-\xi, \lambda) \cos \left(s, x_{m}\right) \mathrm{d} x+ \\
+ & \frac{\lambda}{\nu} \int_{D} \frac{\partial \tilde{u}_{i}(x, \lambda)}{\partial x_{m}} U(x-\xi, \lambda) \mathrm{d} x= \\
= & \int_{D} F_{i}(x, \lambda) \frac{\partial U(x-\xi, \lambda)}{\partial x_{m}} \mathrm{~d} x, \quad(i, m=1,2,3),
\end{aligned}
$$


where $s$ is the outside orthogonal vector on the boundary of $\gamma$. Thus:

$$
\begin{aligned}
& \sum_{k=1}^{3} \int_{\gamma} \frac{\partial \tilde{u}_{i}(x, \lambda)}{\partial x_{k}} \frac{\partial U(x-\xi, \lambda)}{\partial x_{m}} \cos \left(s, x_{k}\right) \mathrm{d} x- \\
- & \sum_{k=1}^{3} \int_{\gamma} \frac{\partial \tilde{u}_{i}(x, \lambda)}{\partial x_{k}} \frac{\partial U(x-\xi, \lambda)}{\partial x_{k}} \cos \left(s, x_{m}\right) \mathrm{d} x+ \\
+ & \sum_{k=1}^{3} \int_{\gamma} \frac{\partial \tilde{u}_{i}(x, \lambda)}{\partial x_{m}} \frac{\partial U(x-\xi, \lambda)}{\partial x_{k}} \cos \left(s, x_{k}\right) \mathrm{d} x- \\
- & \int_{D} \frac{\partial \tilde{u}_{i}(x, \lambda)}{\partial x_{m}} \Delta_{x} U(x-\xi, \lambda) \mathrm{d} x- \\
- & \frac{\lambda}{\nu} \int_{\gamma} \tilde{u}_{i}(x, \lambda) U(x-\xi, \lambda) \cos \left(s, x_{m}\right) \mathrm{d} x+ \\
+ & \frac{\lambda}{\nu} \int_{D} \frac{\partial \tilde{u}_{i}(x, \lambda)}{\partial x_{m}} U(x-\xi, \lambda) \mathrm{d} x= \\
= & \int_{D} F_{i}(x, \lambda) \frac{\partial U(x-\xi, \lambda)}{\partial x_{m}} \mathrm{~d} x .
\end{aligned}
$$

Now by using $(* *)$, we obtain:

$$
\begin{aligned}
& \sum_{k=1}^{3} \int_{\gamma}\left[\frac{\partial \tilde{u}_{i}(x, \lambda)}{\partial x_{k}} \frac{\partial U(x-\xi, \lambda)}{\partial x_{m}}+\right. \\
& \left.+\frac{\partial \tilde{u}_{i}(x, \lambda)}{\partial x_{m}} \frac{\partial U(x-\xi, \lambda)}{\partial x_{k}}\right] \cos \left(s, x_{k}\right) \mathrm{d} x- \\
& -\int_{\gamma}\left[\sum_{k=1}^{3} \frac{\partial \tilde{u}_{i}(x, \lambda)}{\partial x_{k}} \frac{\partial U(x-\xi, \lambda)}{\partial x_{k}}+\right. \\
& \left.+\frac{\lambda}{\nu} \tilde{u}_{i}(x, \lambda) U(x-\xi, \lambda)\right] \cos \left(s, x_{m}\right) \mathrm{d} x- \\
& -\int_{D} F_{i}(x, \lambda) \frac{\partial U(x-\xi, \lambda)}{\partial x_{m}} \mathrm{~d} x= \begin{cases}\frac{\partial \tilde{u}_{i}(\xi, \lambda)}{\partial \xi_{m}}, & \xi \in D, \\
\frac{1}{2} \frac{\partial \tilde{u}_{i}(\xi, \lambda)}{\partial \xi_{m}}, & \xi \in \gamma .\end{cases}
\end{aligned}
$$


Hence:

$$
\begin{aligned}
& \int_{0}^{1} \mathrm{~d} x_{2} \int_{0}^{1} \mathrm{~d} x_{3}\left[\frac{\partial \tilde{u}_{i}(x, \lambda)}{\partial x_{1}} \frac{\partial U(x-\xi, \lambda)}{\partial x_{m}}+\right. \\
& \left.+\frac{\partial \tilde{u}_{i}(x, \lambda)}{\partial x_{m}} \frac{\partial U(x-\xi, \lambda)}{\partial x_{1}}\right]_{x_{1}=1}- \\
& -\int_{0}^{1} \mathrm{~d} x_{2} \int_{0}^{1} \mathrm{~d} x_{3}\left[\frac{\partial \tilde{u}_{i}(x, \lambda)}{\partial x_{1}} \frac{\partial U(x-\xi, \lambda)}{\partial x_{m}}+\right. \\
& \left.+\frac{\partial \tilde{u}_{i}(x, \lambda)}{\partial x_{m}} \frac{\partial U(x-\xi, \lambda)}{\partial x_{1}}\right]_{x_{1}=0}+ \\
& +\int_{0}^{1} \mathrm{~d} x_{1} \int_{0}^{1} \mathrm{~d} x_{3}\left[\frac{\partial \tilde{u}_{i}(x, \lambda)}{\partial x_{2}} \frac{\partial U(x-\xi, \lambda)}{\partial x_{m}}+\right. \\
& \left.+\frac{\partial \tilde{u}_{i}(x, \lambda)}{\partial x_{m}} \frac{\partial U(x-\xi, \lambda)}{\partial x_{2}}\right]_{x_{2}=1}- \\
& -\int_{0}^{1} \mathrm{~d} x_{1} \int_{0}^{1} \mathrm{~d} x_{3}\left[\frac{\partial \tilde{u}_{i}(x, \lambda)}{\partial x_{2}} \frac{\partial U(x-\xi, \lambda)}{\partial x_{m}}+\right. \\
& \left.+\frac{\partial \tilde{u}_{i}(x, \lambda)}{\partial x_{m}} \frac{\partial U(x-\xi, \lambda)}{\partial x_{2}}\right]_{x_{2}=0}+ \\
& +\int_{0}^{1} \mathrm{~d} x_{1} \int_{0}^{1} \mathrm{~d} x_{2}\left[\frac{\partial \tilde{u}_{i}(x, \lambda)}{\partial x_{3}} \frac{\partial U(x-\xi, \lambda)}{\partial x_{m}}+\right. \\
& \left.+\frac{\partial \tilde{u}_{i}(x, \lambda)}{\partial x_{m}} \frac{\partial U(x-\xi, \lambda)}{\partial x_{3}}\right]_{x_{3}=1}- \\
& -\int_{0}^{1} \mathrm{~d} x_{1} \int_{0}^{1} \mathrm{~d} x_{2}\left[\frac{\partial \tilde{u}_{i}(x, \lambda)}{\partial x_{3}} \frac{\partial U(x-\xi, \lambda)}{\partial x_{m}}+\right. \\
& \left.+\frac{\partial \tilde{u}_{i}(x, \lambda)}{\partial x_{m}} \frac{\partial U(x-\xi, \lambda)}{\partial x_{3}}\right]_{x_{3}=0}- \\
& -\int_{0}^{1} \int_{0}^{1} \frac{\mathrm{d} x_{1} \mathrm{~d} x_{2} \mathrm{~d} x_{3}}{\mathrm{~d} x_{m}}\left[\sum_{k=1}^{3} \frac{\partial \tilde{u}_{i}(x, \lambda)}{\partial x_{k}} \frac{\partial U(x-\xi, \lambda)}{\partial x_{k}}+\right. \\
& \left.+\frac{\lambda}{\nu} \tilde{u}_{i}(x, \lambda) U(x-\xi, \lambda)\right]_{x_{m}=1}+ \\
& +\int_{0}^{1} \int_{0}^{1} \frac{\mathrm{d} x_{1} \mathrm{~d} x_{2} \mathrm{~d} x_{3}}{\mathrm{~d} x_{m}}\left[\sum_{k=1}^{3} \frac{\partial \tilde{u}_{i}(x, \lambda)}{\partial x_{k}} \frac{\partial U(x-\xi, \lambda)}{\partial x_{k}}+\right. \\
& \left.+\frac{\lambda}{\nu} \tilde{u}_{i}(x, \lambda) U(x-\xi, \lambda)\right]_{x_{m}=0}- \\
& -\int_{D} F_{i}(x, \lambda) \frac{\partial U(x-\xi, \lambda)}{\partial x_{m}} \mathrm{~d} x= \begin{cases}\frac{\partial \tilde{u}_{i}(\xi, \lambda)}{\partial \xi_{m}}, & \xi \in D, \\
\frac{1}{2} \frac{\partial \tilde{u}_{i}(\xi, \lambda)}{\partial \xi_{m}}, & \xi \in \gamma,\end{cases}
\end{aligned}
$$$$
(i, m=1,2,3) \text {. }
$$ 
Remark 2.2. Contrary to Remark 2.1, here some integral are not computable, but in any case, we obtain many relations from (18), which will be our necessary conditions.

Now from (17), we can see that in the case $x=\xi$ :

$$
\frac{\partial U(x-\xi, \lambda)}{\partial x_{m}}=\frac{x_{m}-\xi_{m}}{4 \pi|x-\xi|^{3}}+\cdots, \quad(m=1,2,3),
$$

where the first term has strong singularity and reminder terms have weak singularity. From (18) and (19), we have"

$$
\begin{gathered}
\left.\frac{\partial \tilde{u}_{i}(\xi, \lambda)}{\partial \xi_{m}}\right|_{\xi_{1}=1}= \\
=\left.\left.2 \int_{0}^{1} \mathrm{~d} x_{2} \int_{0}^{1} \mathrm{~d} x_{3} \frac{\partial \tilde{u}_{i}(x, \lambda)}{\partial x_{1}}\right|_{x_{1}=1} \frac{U(x-\xi, \lambda)}{\partial x_{m}}\right|_{x_{1}=1, \xi_{1}=1}- \\
-\left.\left.2 \sum_{k=1}^{3} \int_{0}^{1} \int_{0}^{1} \frac{\mathrm{d} x_{1} \mathrm{~d} x_{2} \mathrm{~d} x_{3}}{\mathrm{~d} x_{m}} \frac{\partial \tilde{u}_{i}(x, \lambda)}{\partial x_{k}}\right|_{x_{m}=1} \frac{U(x-\xi, \lambda)}{\partial x_{k}}\right|_{x_{m}=1, \xi_{1}=1}+ \\
+\cdots, \quad(i, m=1,2,3) .
\end{gathered}
$$

If $m=1$ and $k=2$ or $k=3$, then there is singularity in the second term of right hand side of (20), and if $m \neq 1$, then there is singularity in the first term of left hand side of (20). Also:

$$
\begin{gathered}
\left.\frac{\partial \tilde{u}_{i}(\xi, \lambda)}{\partial \xi_{m}}\right|_{\xi_{1}=0}= \\
=-\left.\left.2 \int_{0}^{1} \mathrm{~d} x_{2} \int_{0}^{1} \mathrm{~d} x_{3} \frac{\partial \tilde{u}_{i}(x, \lambda)}{\partial x_{1}}\right|_{x_{1}=0} \frac{U(x-\xi, \lambda)}{\partial x_{m}}\right|_{x_{1}=0, \xi_{1}=0}+ \\
+\left.\left.2 \sum_{k=1}^{3} \int_{0}^{1} \int_{0}^{1} \frac{\mathrm{d} x_{1} \mathrm{~d} x_{2} \mathrm{~d} x_{3}}{\mathrm{~d} x_{m}} \frac{\partial \tilde{u}_{i}(x, \lambda)}{\partial x_{k}}\right|_{x_{m}=0} \frac{U(x-\xi, \lambda)}{\partial x_{k}}\right|_{x_{m}=0, \xi_{1}=0}+ \\
+\cdots \quad(i, m=1,2,3) ; \\
\left.\frac{\partial \tilde{u}_{i}(\xi, \lambda)}{\partial \xi_{m}}\right|_{\xi_{2}=1}=\quad \\
\left.\left.\int_{0}^{1} \mathrm{~d} x_{1} \int_{0}^{1} \mathrm{~d} x_{3} \frac{\partial \tilde{u}_{i}(x, \lambda)}{\partial x_{2}}\right|_{x_{2}=1} \frac{U(x-\xi, \lambda)}{\partial x_{m}}\right|_{x_{2}=1, \xi_{2}=1-} \\
-\left.\left.2 \sum_{k=1}^{3} \int_{0}^{1} \int_{0}^{1} \frac{\mathrm{d} x_{1} \mathrm{~d} x_{2} \mathrm{~d} x_{3}}{\mathrm{~d} x_{m}} \frac{\partial \tilde{u}_{i}(x, \lambda)}{\partial x_{k}}\right|_{x_{m}=1} \frac{U(x-\xi, \lambda)}{\partial x_{k}}\right|_{x_{m}=1, \xi_{2}=1}+ \\
+\cdots, \quad(i, m=1,2,3) ;
\end{gathered}
$$




$$
\begin{aligned}
& \left.\frac{\partial \tilde{u}_{i}(\xi, \lambda)}{\partial \xi_{m}}\right|_{\xi_{2}=0}= \\
& =-\left.\left.2 \int_{0}^{1} \mathrm{~d} x_{1} \int_{0}^{1} \mathrm{~d} x_{3} \frac{\partial \tilde{u}_{i}(x, \lambda)}{\partial x_{2}}\right|_{x_{2}=0} \frac{U(x-\xi, \lambda)}{\partial x_{m}}\right|_{x_{2}=0, \xi_{2}=0}+ \\
& +\left.\left.2 \sum_{k=1}^{3} \int_{0}^{1} \int_{0}^{1} \frac{\mathrm{d} x_{1} \mathrm{~d} x_{2} \mathrm{~d} x_{3}}{\mathrm{~d} x_{m}} \frac{\partial \tilde{u}_{i}(x, \lambda)}{\partial x_{k}}\right|_{x_{m}=0} \frac{U(x-\xi, \lambda)}{\partial x_{k}}\right|_{x_{m}=0, \xi_{2}=0}+ \\
& +\cdots, \quad(i, m=1,2,3) \text {; } \\
& \left.\frac{\partial \tilde{u}_{i}(\xi, \lambda)}{\partial \xi_{m}}\right|_{\xi_{3}=1}= \\
& \left.\left.2 \int_{0}^{1} \mathrm{~d} x_{1} \int_{0}^{1} \mathrm{~d} x_{2} \frac{\partial \tilde{u}_{i}(x, \lambda)}{\partial x_{3}}\right|_{x_{3}=1} \frac{U(x-\xi, \lambda)}{\partial x_{m}}\right|_{x_{3}=1, \xi_{3}=1}- \\
& -\left.\left.2 \sum_{k=1}^{3} \int_{0}^{1} \int_{0}^{1} \frac{\mathrm{d} x_{1} \mathrm{~d} x_{2} \mathrm{~d} x_{3}}{\mathrm{~d} x_{m}} \frac{\partial \tilde{u}_{i}(x, \lambda)}{\partial x_{k}}\right|_{x_{m}=1} \frac{U(x-\xi, \lambda)}{\partial x_{k}}\right|_{x_{m}=1, \xi_{3}=1}+ \\
& +\cdots, \quad(i, m=1,2,3) \text {; } \\
& \left.\frac{\partial \tilde{u}_{i}(\xi, \lambda)}{\partial \xi_{m}}\right|_{\xi_{3}=0}= \\
& =-\left.\left.2 \int_{0}^{1} \mathrm{~d} x_{1} \int_{0}^{1} \mathrm{~d} x_{2} \frac{\partial \tilde{u}_{i}(x, \lambda)}{\partial x_{3}}\right|_{x_{3}=0} \frac{U(x-\xi, \lambda)}{\partial x_{m}}\right|_{x_{3}=0, \xi_{3}=0}+ \\
& +\left.\left.2 \sum_{k=1}^{3} \int_{0}^{1} \int_{0}^{1} \frac{\mathrm{d} x_{1} \mathrm{~d} x_{2} \mathrm{~d} x_{3}}{\mathrm{~d} x_{m}} \frac{\partial \tilde{u}_{i}(x, \lambda)}{\partial x_{k}}\right|_{x_{m}=0} \frac{U(x-\xi, \lambda)}{\partial x_{k}}\right|_{x_{m}=0, \xi_{3}=0}+ \\
& +\cdots, \quad(i, m=1,2,3) \text {. }
\end{aligned}
$$

By using Laplace transformation on the boundary condition (12), we obtain

$$
\left\{\begin{array}{rl}
\tilde{u}_{i}\left(0, x_{2}, x_{3}, \lambda\right) & =\tilde{u}_{i}\left(1, x_{2}, x_{3}, \lambda\right), \\
\tilde{u}_{i}\left(x_{1}, 0, x_{3}, \lambda\right) & =\tilde{u}_{i}\left(x_{1}, 1, x_{3}, \lambda\right), \\
\tilde{u}_{i}\left(x_{1}, x_{2}, 0, \lambda\right) & =\tilde{u}_{i}\left(x_{1}, x_{2}, 1, \lambda\right), \\
\left.\frac{\partial \tilde{u}_{i}(x, \lambda)}{\partial x_{m}}\right|_{x_{k}=0}=\left.\frac{\partial \tilde{u}_{i}(x, \lambda)}{\partial x_{m}}\right|_{x_{k}=1},
\end{array} \quad(i, m, k=1,2,3) .\right.
$$

From (19) and (26) we see that by addition of (20) and (21), (22) and (23), (24) and (25), the parts which have strong singularity will be delete, so in the terms of:

$$
\left.\frac{\partial \tilde{u}_{i}(\xi, \lambda)}{\partial \xi_{m}}\right|_{\xi_{k}=0}+\left.\frac{\partial \tilde{u}_{i}(\xi, \lambda)}{\partial \xi_{m}}\right|_{\xi_{k}=1}=\cdots \quad(i, m, k=1,2,3),
$$

there is not the strong singularity. Now, we can see from $(16)$ that $\tilde{u}_{i}(\xi, \lambda)$ is depend to the boundary values of $u_{i}(\xi, \lambda),\left.\frac{\partial \tilde{u}_{i}(x, \lambda)}{\partial x_{k}}\right|_{x_{k}=0}$ and $\left.\frac{\partial u_{i}(x, \lambda)}{\partial x_{k}}\right|_{x_{k}=1}$, $(i, k=1,2,3)$, whenever $\xi \in D$. By using $(26)$, boundary values of $\tilde{u}_{i}(x, \lambda)$ 
and (16), we obtain three second type Fredholm integral equations which their kernels have weak singularity. Similarly by using of (26), (27), the boundary values $\left.\frac{\partial \tilde{u}_{i}(x, \lambda)}{\partial x_{k}}\right|_{x_{k}=0}$ and $\left.\frac{\partial u_{i}(x, \lambda)}{\partial x_{k}}\right|_{x_{k}=1},(i, k=1,2,3)$, we obtain six second type Fredholm integral equations which their kernels have weak singularity.

Remark 2.3. In the above equations which be used, in addition to values:

$$
\left.\frac{\partial \tilde{u}_{i}(x, \lambda)}{\partial x_{k}}\right|_{x_{k}=0}, \quad(k=1,2,3)
$$

there are also the values:

$$
\left.\frac{\partial \tilde{u}_{i}(x, \lambda)}{\partial x_{m}}\right|_{x_{k}=0} \quad(m, k=1,2,3 ; m \neq k) .
$$

Since:

$$
\left.\frac{\partial \tilde{u}_{i}(x, \lambda)}{\partial x_{m}}\right|_{x_{k}=0}=\frac{\partial}{\partial x_{m}}\left[\left.\tilde{u}_{i}(x, \lambda)\right|_{x_{k}=0}\right]
$$

we could calculate the integrals of these functions by integration part by part, and so we will differentiate respect to $x_{m}$ of the fundamental solutions which are in side of the values $\frac{\partial \tilde{u}_{i}(x, \lambda)}{\partial x_{m}}$. Hence, strong singularity will not appear.

Note that it follows from periodicity condition of $\tilde{u}_{i}(x, \lambda)$ that all values of $\tilde{u}_{i}(x, \lambda)$ are equal on parallel faces of the unit cube, and so all values of $\tilde{u}_{i}(x, \lambda)$ are equal on vertices of the unit cube.

If $\xi$ is choose on common axes of faces of the unit cube, then from (16) and (18), we obtain integro-differential systems which will be our necessary conditions. By using (26) and (27), we could calculate the values:

$$
\left.\frac{\partial \tilde{u}_{i}(x, \lambda)}{\partial x_{m}}\right|_{x_{k}=0} \quad(m, k=1,2,3 ; m \neq k),
$$

and then by integration respect to $x_{m}$ for the functions $\left.\tilde{u}_{i}(x, \lambda)\right|_{x_{k}=0},(k=$ $1,2,3)$, we will obtain an integral equations system in which the kernels have weak singularity.

If we write the unknowns of this equations system as the form:

$$
\begin{aligned}
& \left(\tilde{u}_{i}\left(0, x_{2}, x_{3}, \lambda\right), \tilde{u}_{i}\left(x_{1}, 0, x_{3}, \lambda\right), \tilde{u}_{i}\left(x_{1}, x_{2}, 0, \lambda\right),\right. \\
& \left.\frac{\partial \tilde{u}_{i}(x, \lambda)}{\partial x_{1}}\right|_{x_{1}=0},\left.\frac{\partial \tilde{u}_{i}(x, \lambda)}{\partial x_{1}}\right|_{x_{2}=0},\left.\frac{\partial \tilde{u}_{i}(x, \lambda)}{\partial x_{1}}\right|_{x_{3}=0}, \\
& \left.\frac{\partial \tilde{u}_{i}(x, \lambda)}{\partial x_{2}}\right|_{x_{1}=0},\left.\frac{\partial \tilde{u}_{i}(x, \lambda)}{\partial x_{2}}\right|_{x_{2}=0},\left.\frac{\partial \tilde{u}_{i}(x, \lambda)}{\partial x_{2}}\right|_{x_{3}=0}, \\
& \left.\left.\frac{\partial \tilde{u}_{i}(x, \lambda)}{\partial x_{3}}\right|_{x_{1}=0},\left.\frac{\partial \tilde{u}_{i}(x, \lambda)}{\partial x_{3}}\right|_{x_{2}=0},\left.\frac{\partial \tilde{u}_{i}(x, \lambda)}{\partial x_{3}}\right|_{x_{3}=0}\right) \equiv \tilde{U}_{i}, \\
& (i=1,2,3),
\end{aligned}
$$


then we could write this system as:

$$
\tilde{U}_{i}=T \tilde{U}_{i}+\tilde{F}_{i}, \quad(i=1,2,3),
$$

where, $\tilde{F}_{i}$ is a column vector of known functions and $T$ is the integral operator in which its kernel tends to zero, whenever:

$$
\lambda \in R_{\sigma}=\{\lambda:-\pi+\sigma \leq \arg \lambda \leq \pi-\sigma\}
$$

and $|\lambda|$ is sufficiently large. Thus, the system (29) has an unique solution.

After solve of the system (29), all functions of (28) will be obtain which by replacement in (26) we will have a non-linear integro-differential system according to the functions $\tilde{u}_{i}(\xi, \lambda)(\xi \in D)$. By solving it we could find the functions $\tilde{u}_{i}(\xi, \lambda)(\xi \in D)$. Now by the inverse Laplace transform, we could obtain the functions $u_{i}(\xi, \tau)(\xi \in D)$ and by periodicity of these functions, we could find the functions $u_{i}(\xi, \tau)$ on $\mathbb{R}^{3}$. Therefore, we could obtain $p(\xi, \tau)$ as the method which is provided in [5].

Theorem 2.4. Suppose that in the relations (8)-(10), $\nu>0, u^{0}$ satisfies in the condition (11) and:

$$
\int_{[0,1]^{3}} u_{i}^{0}(x) \mathrm{d} x=0,
$$

for all index $i(1 \leq i \leq 3)$. Then, the problem $B$ has an unique solution on the cube which satisfies in the conditions (11) and (12).

Now, we could obtain the unique solution of the problem $B$ on $\mathbb{R}^{3}$ by periodic extension the above unique solution.

\section{REFERENCES}

[1] N. Aliev, M. Jahanshahi, Sufficient Conditions for Reduction of the BVP Including a Mixed PDE with Non-local Boundary Conditions to Fredholm Integral Equations, Int. J. Math. Educ. Sci. Technol., Vol. 28, No. 3 (1997), 419-425.

[2] N. Aliev, S.M. Hosseini, An Analysis of a Parabolic Problem with General (Non-local and Global) Supplementary Linear Conditions I, Italian J. of Pure and Appl. Math., No. 12 (2002), 143-154.

[3] N. Aliev, S.M. Hosseini, An Analysis of a Parabolic Problem with General (Non-local and Global) Supplementary Linear Conditions II, Italian J. of Pure and Appl. Math., No. 13 (2003), 115-127.

[4] N. Aliev, Sh. Rezapour, M. Jahanshahi, On Fefferman's Non-existence Problems, Mathematica Morvica, Vol. 11 (2007), 1-7.

[5] N. Aliev, Sh. Rezapour, M. Jahanshahi, A Mixed Problem for Navier-Stokes System, Mathematica Morvica, Vol. 12(2) (2008), 1-15.

[6] V.I. Arnold, Ordinary Differential Equations, Springer-Verlag, 1992.

[7] A.V. Bitsadze, Boundary Value Problems including Second Order Elliptic Equations, North-Holland Publication, First edition, 1968.

[8] R. Courant, D. Hilbert, Methods of Mathematical Physics, Vol. II, John-Wiely, 1953.

[9] A.A. Dezin, Partial Differential Equations, Springer-Verlag, 1987. 
[10] A.A. Dorodnichen, On the Approach to the Solution of Boundary Value Problems for Differential Equations (Computational Experiment), Contemporary Problems of Applied Mathematics and Mathematical Physics, Moscow, 1988, 31-42 (in Russian).

[11] S.M. Hosseini, N. Aliev, A Mixed Parabolic with a Non-local and Global Linear Conditions, J. Sci. I. R. Iran, Vol. 11, No. 3 (2000), 233-237.

[12] S.M. Hosseini, N. Aliev, Sufficient Conditions for the Reduction of the BVP for PDE with Non-local and Global Boundary Conditions to Fredholm Integral Equations (on a Rectangular Domain, Appl. Math. and Computation, 147 (2004), 669-685.

[13] G. Kavei, N. Aliev, An Analytical Methods to the Solution of the Time-dependent Schrodinger Equation Using Half Cylinder Space System-I, Bull. of Pure and Appl. Sciences, Vol. 16E, No. 2 (1997), 253-263.

[14] G. Kavei, N. Aliev, The Existence and Uniqueness of the Solution of the Spectral Problem II, J. Sci. I. R. Iran, Vol. 10, No. 4 (1999), 252-258.

[15] A.K. Pani, G. Fairweather, $H^{1}$-Galerkin Mixed Finite Element Methods for Parabolic Partial Integro-differential Equations, IMA J. Numer. Anal., Vol. 22, No. 2 (2002), 231-252.

[16] M.L. Rasulov, Methods of Contour Inegration, North-Holland, 1967.

[17] S.G. Samko, A.A. Kilbas, O.I. Marichev, Integrals and Derivatives of Fractional Order and Some of their Applications, (in Russian), Nauka and Technika, Minsk, 1987.

[18] S. Shahmorad, Numerical solution of the general form linear FredholmÜVolterra integro-differential equations by the Tau method with an error estimation, Applied Mathematics and Computation, Vol. 167, Issue 2 (2005), 1418-1429.

[19] V.S. Vladimirov, Equations of Mathematical Physics, Mir Publisher, Moscow, 1984.

[20] V.S. Vladimirov, Generalized Functions in Mathematical Physics, Mir Publisher, Moscow, 1979.

\section{N. Aliev}

Department of Mathematics

Azarbaidjan University of Tarbiat Moallem 53714-161 Azarshahr, TABriz

IRAN

\section{Sh. Rezapour}

Department of Mathematics

Azarbaidjan University of Tarbiat Moallem 53714-161 Azarshahr, Tabriz

IRAN

E-mail address: sh.rezapour@azaruniv.edu

\section{JAHANSHAHI}

Department of Mathematics

Azarbaidjan University of Tarbiat Moallem 53714-161 Azarshahr, Tabriz

IRAN

E-mail address: jahan_m15@yahoo.com 